\title{
Oculomotor neuropathy following tetanus toxoid injection
}

Sir,

Anti-tetanus toxoid, a widely used prophylactic 
conduct in patients with open wounds, is considered to be very safe. However, there have been several reports of peripheral neuropathy occurring hours to weeks after its injection; the estimated incidence is 0.4 per million administered doses of the toxoid. ${ }^{[1]}$ These reports are consistent with neuropathy as a manifestation of immune complex disease. ${ }^{[2]}$ There are very few reports of cranial nerve palsy following tetanus toxoid vaccination; the nerves involved include the optic, facial and auditory nerves..$^{[3-5]}$ Most of the reported cranial nerve palsies were reversible and developed within a few days of the vaccination.

A 16-year-old girl with no previous remarkable medical illnesses presented to our clinic complaining of blurred vision in her right eye that started a few hours after she had received an injection of anti-tetanus toxoid for a leg wound sustained in an injury. She also experienced mild right frontal headache. On the day following the injection she suffered double vision, especially when looking down and to the left. Her family noticed abnormal alignment of her eyes and mild drooping of the right eyelid.

On examination there were right eyelid ptosis and anisocoria ( 6 and $3 \mathrm{~mm}$ right and left pupil, respectively); the right pupil reacted weakly to light. This was associated with weak upward, downward and inward movement of the right eye.

The patient was admitted to look for the cause of her oculomotor nerve palsy. Laboratory results including a complete blood count, erythrocyte sedimentation rate, C-reactive protein and kidney- and liver function tests were all within the normal range. Magnetic resonance imaging (MRI) and MR angiography (MRA) returned no significant findings [Figure 1]. Although we recommended digital subtraction angiography (DSA) to rule out third cranial nerve compression by an internal carotid-posterior communicating artery aneurysm her family refused the procedure. Lumbar puncture revealed normal opening pressure; cerebrospinal fluid (CSF) findings were normal (2 lymphocytes $/ \mathrm{mm}^{3}$, PMN 0, protein $38 \mathrm{mg} / \mathrm{dl}$, glucose $75 \mathrm{mg} / \mathrm{dl}$ ) and there was no oligoclonal band. We interpreted the clinical findings to be reflective of a rare toxic neuropathy secondary to tetanus toxoid injection and she was treated with intravenous methylprednisolone $500 \mathrm{mg}$ twice a day for 7 days.

During her hospital stay she showed significant symptom improvement and her third nerve palsy cleared on day 7 of the treatment.

Compression of the subarachnoid segment of the oculomotor nerve is the most common cause of single complete third nerve palsy. Pathologies affecting other segments of the third nerve (nuclear, midbrain, cavernous orbital) are usually associated with long tracts, partial third cranial nerve palsy and/or the involvement
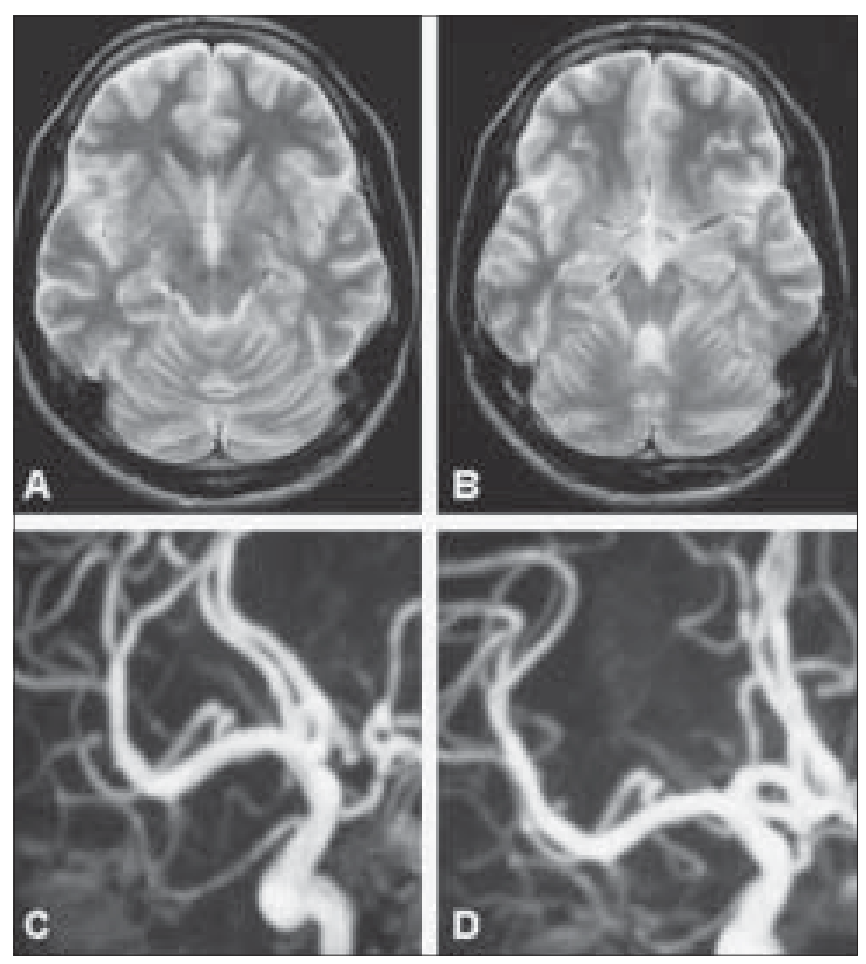

Figure 1: Axial T2-weighted MRI at the level of the midbrain (A) and interpeduncular cistern (B), showing no pathological changes at these levels. (C, D) MRA of the cerebral vessels showing no apparent aneurysmal dilatation

of other cranial nerves. The most common lesion to affect the third cranial nerve in the subarachnoid space is compression by aneurysm, basal meningeal infection, neoplastic infiltration and miscellaneous inflammatory lesions. The absence of meningeal irritation signs and the normal imaging and CSF studies in our patient made a diagnosis of aneurysmal compression, neoplastic or inflammatory infiltration of the third nerve unlikely. Instead, the fact that her third nerve palsy developed shortly after tetanus toxoid injection made us suspect that her symptoms were related to the tetanus vaccination.

Our review of the literature disclosed few reports of cranial nerve palsy after the injection of tetanus toxoid [Table 1]. As in our patient, the cranial nerve palsy in the reported cases started a few days after injection and resolved within a few weeks either with or without treatment.

To our knowledge, this is the first case of third cranial nerve palsy following the injection of tetanus toxoid. The exact mechanism(s) by which tetanus toxoid produces cranial nerve palsy remains unknown, however, it may be the direct toxic effect of the vaccine or an immune-mediated reaction. Risk factors and patients susceptible to this complication are also unidentified. The administration of steroids appears to be very helpful to hasten recovery, as was the case in our patient. 
Table 1: Reported cases of cranial nerve palsy following tetanus toxoid injection

\begin{tabular}{ll}
\hline Author, year & Involved cranial nerve \\
Kharoubi, 2005 & Facial nerve \\
Burkhard et al., 2001 & Optic nerve \\
Basek, 1958 & Vagus nerve (recurrent laryngeal nerve) \\
Bauer et al., 1957[7] & Vagus nerve (recurrent laryngeal nerve) \\
Cutter, 1936 & Auditory nerve \\
Present report, 2006 & Oculomotor nerve \\
\hline
\end{tabular}

\section{Jamous Mohammad, Al-Hayek Kefah,} Hani Abdel Aziz

Department of Neuroscience, Faculty of Medicine, Jordan University of Science and Technology, Irbid, Jordan.

E-mail: mojamous@yahoo.com

\section{References}

1. Holliday PL, Bauer RB. Polyradiculoneuritis secondary to immunization with tetanus and diphtheria toxoid. Arch Neurol 1983;40:56-7.

2. Rutledge SL, Snead OC. Neurologic complications of immunizations. J Pediatr 1986;109:917-23.

3. Burkhard C, Choi M, Wilhelm H. Optic neuritis as a complication in preventive tetanus-diphtheria-poliomyelitis vaccination: A case report. Klin Monatsbl Augenheilkd 2001;218:51-4 (in German).

4. Cutter RD. Auditory nerve involvement after tetanus antitoxin: First reported case. JAMA 1936;21:1006-7.

5. Kharoubi S. Peripheral facial paralysis associated with anti-tetanic serotherapy: A case report. Rev Neurol (Paris) 2005;161:469-71 (in French).

6. Basek F. Unilateral paralysis of vocal cords following administration of tetanus antitoxin. Laryngoscope 1958;88:805-7.

7. Bauer F, Ellis W. Paralysis of the recurrent laryngeal nerve following injection of antitetanus serum. J Laryngol 1957;71:131-3.

Accepted on 07-01-2008 